\title{
Does Knee Arthroscopy for Treatment of Meniscal Damage with Osteoarthritis Delay Knee Replacement Compared to Physical Therapy Alone?
}

\author{
Ronald A. Navarro, MD, Annette L. Adams, $\mathrm{PhD}^{*}$, Charles C. Lin, $\mathrm{MD}^{\dagger}$, John Fleming, $\mathrm{MD}^{\ddagger}$, \\ Ivan A. Garcia, $\mathrm{MD}^{\S}$, Janet Lee, $\mathrm{MS}^{*}$, Mary Helen Black, $\mathrm{PhD}^{*}$ \\ Department of Orthopaedic Surgery, Kaiser Permanente South Bay Medical Center, Harbor City, CA, \\ ${ }^{*}$ Department of Research and Evaluation, Kaiser Permanente Southern California, Pasadena, CA, \\ ${ }^{\dagger}$ Department of Orthopedic Surgery, NYU Langone Orthopedic Hospital, New York, NY, \\ ${ }^{\ddagger}$ Torrance Orthopaedic and Sports Medicine Group, Torrance, CA, \\ ${ }^{\S}$ Kaiser Permanente Baldwin Park Medical Center, Baldwin Park, CA, USA
}

Background: To determine patient factors that lead to treatment of meniscal tears with osteoarthritis (OA) with knee arthroscopy (KA) or physical therapy only (PT-only); and to assess differences in clinical outcomes including the time to knee arthroplasty.

Methods: Patients aged $\geq 45$ years with OA at meniscal tear diagnosis were followed up from the date of surgery (KA) or first PT visit (PT-only) until partial/total knee replacement surgery, death, disenrollment, or end of study. Demographic and clinical characteristics were compared and used to derive propensity scores. A Cox proportional hazards model was used to estimate the risk of knee replacement surgery and greater healthcare utilization associated with KA vs. PT-only.

Results: Among 7,026 patients (KA, 69\%; PT-only, 31\%), 27\% had partial or total knee replacement surgery during follow-up. PT-only patients were older and more likely to be women and had more comorbidities. After accounting for differences between groups, the cumulative incidence of knee replacement was modestly but significantly higher for those who received KA than those who underwent PT-only (hazard ratio, 1.30; 95\% confidence interval, 1.17-1.44; $p<0.001$ ), although there was no significant difference in health service utilization, narcotic medication dispenses, or knee injections after initiating treatment.

Conclusions: For patients with meniscal damage complicated by OA, those who underwent KA were $30 \%$ more likely to have partial or total knee replacement surgery at any given time than those who had PT alone.

Keywords: Meniscus tear, Osteoarthritis, Knee arthroscopy

The prevalence of radiographically defined knee osteoarthritis (OA) in the United States has been shown to range from $15 \%$ to $50 \%$ among individuals $\geq 45$ years of age. ${ }^{1,2)}$ Findings of meniscal damage are also common, with a

Received July 22, 2019; Accepted November 25, 2019

Correspondence to: Ronald A. Navarro, MD

Department of Orthopaedic Surgery, Kaiser Permanente South Bay Medical Center, Coastline MOB 25821 S. Vermont Ave., Harbor City, CA 90710, USA Tel: +1-424-251-7344, Fax: +1-424-251-7284

E-mail: Ronald.A.Navarro@kp.org
$76 \%$ prevalence in asymptomatic individuals $\geq 50$ years old without radiographic evidence of knee arthritis, and even more frequent in patients with $\mathrm{OA}$, with prevalence up to $91 \%$ in symptomatic patients. ${ }^{3-5)}$

Knee arthroscopy (KA) is one of the most commonly performed orthopedic procedures and its use continues to increase ${ }^{6-8)}$ While previous randomized controlled trials have shown no benefit of KA in patients with $\mathrm{OA},{ }^{9,10)}$ these trials did not focus on knee OA complicated by a meniscal tear, and therefore, could not inform clinical decisionmaking regarding KA for meniscal damage in the setting

Copyright (C) 2020 by The Korean Orthopaedic Association 
of OA. Subsequent randomized controlled trials for degenerative meniscal pathologies found no difference in pain relief or functional status between patients treated with $\mathrm{KA}$ and physical therapy (PT) and those who underwent $\mathrm{PT}$ alone. ${ }^{11-13)}$ However, KA is still commonly viewed as a temporary measure that can achieve pain relief and delay arthroplasty. ${ }^{7,14)}$

The purpose of this study of nearly 12,000 patients with meniscal damage in the setting of OA was to evaluate the demographic and clinical patient factors that lead to the treatment choice of KA compared to PT-only and to assess whether time to arthroplasty was significantly different for patients who underwent KA vs. PT-only. We hypothesized that patients who underwent KA would be more likely to have partial or total knee replacement surgery at any given time than those who had PT alone.

\section{METHODS}

\section{Study Population}

From 2002 to 2006, 74,089 patients were diagnosed with mild-to-moderate OA and 14,731 of those were concomitantly diagnosed with a meniscal tear. Of the 11,594 who met eligibility criteria, 4,883 underwent KA, 2,143 had PT alone, and 4,568 underwent pain management only, without KA or PT (Fig. 1). Among 7,026 patients who had KA (69\%) or PT-only (31\%), 1,884 (26.8\%) had partial or total knee replacement surgery during follow-up. This retrospective cohort study was conducted within the Kaiser Permanente Southern California (KPSC), an integrated healthcare system with a racially and socioeconomically diverse membership of approximately 4.5 million people who are broadly representative of the underlying population. The Institutional Review Board of KPSC approved the study (IRB No. 10184).

The study cohort comprised KPSC members who met the following eligibility criteria in the period between January 1, 2002 and December 31, 2006: patients who (1) were active members and aged $\geq 45$ years, (2) had $\geq 2$ years of health plan membership duration and no prior history of any knee surgery, (3) had an X-ray of the knee and subsequent diagnosis of mild to moderate knee OA, and (4) after X-ray, had a magnetic resonance imaging (MRI) and subsequent diagnosis of medial or lateral meniscal tear. Treatment with PT and/or KA was assessed among patients meeting these inclusion criteria through December 31, 2008. It was assumed that time to outcome after KA was due to the surgery and not any prior or subsequent PT. Patients who met the inclusion criteria but never received either PT or arthroscopy were excluded from the analysis. Pregnant women and patients whose meniscal damage was complicated by other tears or defects identified by MRI, such as presence of loose bodies or anterior/posterior cruciate ligament tears, were also excluded.

\section{Cohort Ascertainment}

Current Procedural Terminology, 4th Edition (CPT4) codes for diagnostic X-ray of the knee (73560, 73562, 73564, 73565) and International Classification of Diseases, Ninth Revision (ICD-9) codes for mild-to-moderate arthritis (715.X6, 715.89) followed by CPT4 codes for MRI for meniscal damage $(73721,73722,73723)$ and ICD-9 codes for meniscal tears (836.0-836.2, 717.0-717.5, 717.9, 718.00) were used in the identification of the cohort, as well as treatment with PT (CPT4: 97001, 97750, 97110, 97112, 97116, 97140) or KA (CPT4: 29868, 29870, 29877, 29880, 29881, 29882, 29883).

\section{Follow-up}

The remaining cohort of patients was followed up from initial treatment (the first PT encounter for the PT-only group, or the date of surgery for the KA group) until one of the following: occurrence of a primary outcome of interest, death, disenrollment from the health plan (allowing for $>90$ day gap in membership), or December 31, 2013 (maximum potential follow-up of 11 years). If a patient in the PT-only group underwent a KA during the period

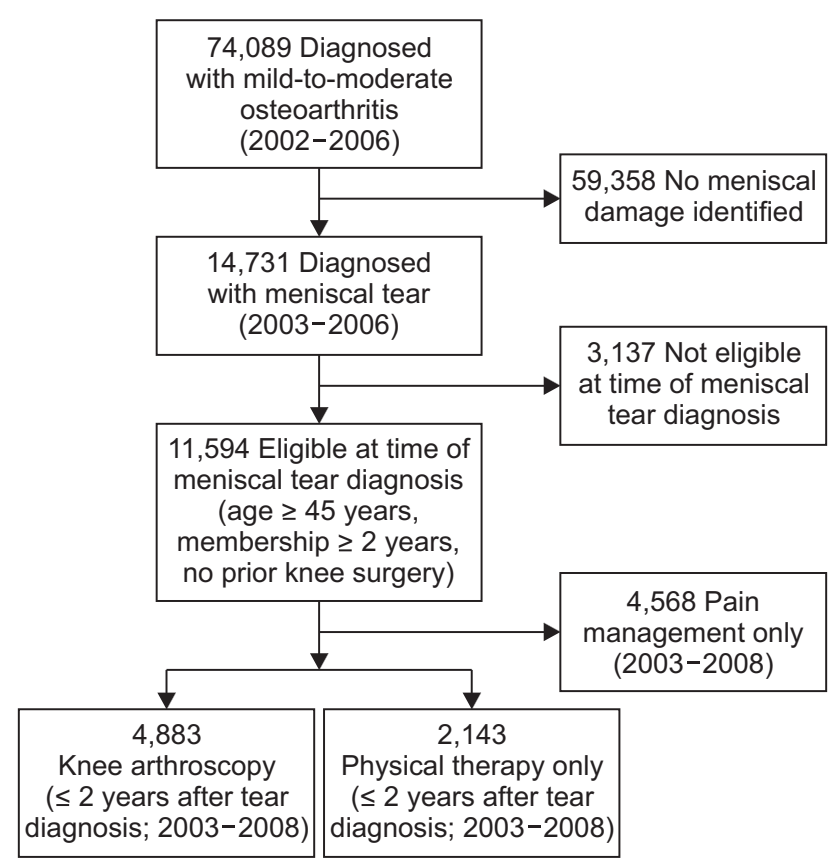

Fig. 1. Cohort ascertainment 
2008-2013, which is in the range of 2 to 10 years after meeting study inclusion criteria, he/she was censored at the date of surgery. The primary outcomes of interest were partial or total knee replacement or knee fusion (identified by CPT4 codes $27440-27447$ and 27580 ). Secondary outcomes included the number of ambulatory visits to the orthopedics department, encounters in the clinics for pain control specific to the knee, pharmacy prescription fills for pain medications, and number of knee injections.

\section{Demographic and Clinical Patient Characteristics}

Patient characteristics at index (date of meniscal tear diagnosis), such as age, sex, race/ethnicity, payer for insurance, census-based education level, household income, comorbidities (for derivation of the Charlson Comorbidity Index), ${ }^{15)}$ body mass index, physical activity, alcohol consumption, and smoking status were extracted. The number of ambulatory office visits, hospitalizations and/ or emergency department visits, both general and knee pain-specific (ICD-9 codes: 719.40, 719.46, 719.49), were collected for the year prior to index and the first year of follow-up.

\section{Data Quality Assessment}

To assess the quality of the coding used to define the cohort and identify treatment and outcomes, we performed a manual chart review of 400 patient records, randomly selected from patients meeting study inclusion criteria: 150 identified as having KA and 250 identified as having PT-only. These proportions were chosen to maximize our ability to detect various forms of treatment for the PTonly group, which were thought to be more challenging to identify and characterize.

\section{Statistical Analysis}

Demographic and clinical variables for the treatment groups were compared by using chi-square test for categorical variables and $t$-tests or Wilcoxon rank-sum tests for continuous variables as appropriate. Cox proportional hazards models were used to estimate the risk of knee replacement or fusion surgery associated with treatment by KA vs. PT-only. Treatment selection was assumed to be categorically fixed and person-time accumulated from date of KA or PT until partial or total knee replacement, knee fusion, death, disenrollment from the health plan, or end of study period, whichever occurred first. Initial treatment choice could be dependent on several pretreatment factors that may also independently increase risk for knee replacement or fusion surgery over time. To correct for this possible bias, logistic regression was used to compute a propensity score as the predicted probability of treatment choice conditional on the set of demographic and clinical pretreatment variables. ${ }^{16,17)}$

Inverse probability of treatment weights (IPTW) were then used to weigh individual observation in the estimation of Cox model parameters. The IPTW was calculated as the inverse of the propensity score and included age, gender, race/ethnicity, education level, assigned medical center at time of tear, comorbidities, indicator for use of MRI, ambulatory office visits and hospitalizations, both general and knee pain-specific in the year prior to tear, and medication use prior to tear. SAS ver. 9.2 (SAS Institute Inc., Cary, NC, USA) was used for statistical analysis. Statistical significance was determined at $p<0.05$.

\section{RESULTS}

Overall, the median (interquartile range: the difference between 75th and 25th percentile) of patient age at initial diagnosis of meniscal tear was 60 years (9), 40\% were men, and the majority were non-Hispanic white $(58 \%)$ or Hispanic (24\%). The majority of patients (54\%) had Charlson Comorbidity scores of 0 and $24 \%$ had scores greater than 2. Additionally, $6 \%$ of patients were current smokers and $63 \%$ never smoked. PT-only patients were older and more likely to be women or African American; they also had a greater comorbidity burden, had fewer outpatient visits for knee pain, and utilized PT more frequently in the year before tear diagnosis. They were also less likely to have used narcotic pain medications or had knee injections in the year prior to meniscal tear diagnosis than patients with KA (Table 1). After propensity score weighting, there was a more balanced distribution of baseline characteristics between patients who received KA and PT. In particular, there was more balance for age, gender, race/ethnicity, Charlson Comorbidity scores, outpatient visits in the year prior to tear, and prior PT sessions. After weighting, the standardized difference was $<0.1$ for all variables.

After accounting for differences between groups, KA patients were no better off than PT-only patients in terms of time to knee replacement surgery (Fig. 2). Total prevalence of knee arthroplasty or fusion was significantly higher for those who received KA (1,396 knee arthroplasty or fusion per 4,883 KA patients [28.6\%]) than those who received PT-only (488 knee arthroplasty or fusion per 2,143 PT-only patients [22.8\%]) (hazard ratio, 1.30; 95\% confidence interval, 1.17-1.44) (Table 2). No statistically significant differences in healthcare utilization for knee pain, narcotic medication dispenses, or knee injections were observed between the groups (Table 3). 
Navarro et al. Knee Arthroscopy to Knee Replacement Time

Clinics in Orthopedic Surgery • Vol. 12, No. 3, $2020 \bullet$ www.ecios.org

Table 1. Demographics, Clinical Characteristics, Healthcare Utilization, and Medication Use

\begin{tabular}{|c|c|c|c|c|}
\hline Variable & $\begin{array}{l}\text { Knee arthroscopy } \\
\qquad(\mathrm{n}=4,883)\end{array}$ & $\begin{array}{l}\text { Physical therapy only } \\
\qquad(n=2,143)\end{array}$ & $\begin{array}{c}\text { Total } \\
(n=7,026)\end{array}$ & $p$-value \\
\hline Age (yr) & & & & $<0.001$ \\
\hline $45-54$ & $1,706(35)$ & $586(27)$ & 2,292 (33) & \\
\hline $55-64$ & 1,848 (38) & $762(36)$ & $2,610(37)$ & \\
\hline $65-74$ & $1,037(21)$ & $550(26)$ & 1,587 (23) & \\
\hline$\geq 75$ & $292(6)$ & $245(11)$ & $537(8)$ & \\
\hline Sex & & & & $<0.001$ \\
\hline Male & $2,175(45)$ & $641(30)$ & $2,816(40)$ & \\
\hline Female & $2,708(55)$ & $1,502(70)$ & $4,210(60)$ & \\
\hline Race/ethnicity & & & & $<0.001$ \\
\hline White & 2,981 (61) & $1,127(53)$ & $4,108(58)$ & \\
\hline Black & $467(10)$ & $317(15)$ & $784(11)$ & \\
\hline Hispanic & $1,147(23)$ & $505(24)$ & 1,652 (24) & \\
\hline Asian Pacific island & $197(4)$ & $107(5)$ & $304(4)$ & \\
\hline Other/unknown & $91(2)$ & 87 (4) & 178 (3) & \\
\hline Insurance type & & & & $<0.001$ \\
\hline Commercial & $3,274(67)$ & $1,265(59)$ & $4,539(65)$ & \\
\hline Medicaid & $47(1)$ & $25(1)$ & $72(1)$ & \\
\hline Medicare & $1,275(26)$ & $748(35)$ & $2,023(29)$ & \\
\hline Private pay & $282(6)$ & $104(5)$ & $386(5)$ & \\
\hline Other & $5(0)$ & $1(0)$ & $6(0)$ & \\
\hline Charlson Comorbidity Index & & & & $<0.001$ \\
\hline 0 & $2,700(55)$ & $1,070(50)$ & $3,770(54)$ & \\
\hline 1 & $1,100(23)$ & $479(22)$ & $1,579(22)$ & \\
\hline$\geq 2$ & $1,083(22)$ & $594(28)$ & $1,677(24)$ & \\
\hline Smoking status & & & & $<0.001$ \\
\hline Current & $352(7)$ & $103(5)$ & $455(6)$ & \\
\hline Former & 1,127 (23) & 472 (22) & 1,599 (23) & \\
\hline Never & $2,979(61)$ & $1,413(66)$ & $4,392(63)$ & \\
\hline Missing & $425(9)$ & $155(7)$ & $580(8)$ & \\
\hline \multicolumn{2}{|c|}{ Outpatient visit for knee pain in year prior to tear diagnosis } & & & $<0.001$ \\
\hline 0 & $1,684(34)$ & $907(42)$ & $2,591(37)$ & \\
\hline 1 & $1,672(34)$ & $688(32)$ & $2,360(34)$ & \\
\hline$\geq 2$ & 1,527 (31) & $548(26)$ & 2,075 (30) & \\
\hline
\end{tabular}


Table 1. Continued

\begin{tabular}{|c|c|c|c|c|}
\hline Variable & $\begin{array}{l}\text { Knee arthroscopy } \\
\quad(n=4,883)\end{array}$ & $\begin{array}{l}\text { Physical therapy only } \\
\qquad(\mathrm{n}=2,143)\end{array}$ & $\begin{array}{c}\text { Total } \\
(\mathrm{n}=7,026)\end{array}$ & $p$-value \\
\hline Physical therapy visit in year prior to tear diagnosis & & & & $<0.001$ \\
\hline 0 & $2,973(61)$ & $1,093(51)$ & $4,066(58)$ & \\
\hline $1-4$ & $1,022(21)$ & $523(24)$ & $1,545(22)$ & \\
\hline$>4$ & $888(18)$ & $527(25)$ & $1,415(20)$ & \\
\hline \multicolumn{5}{|c|}{ Pharmaceutical pain management in year prior to tear diagnosis } \\
\hline Patient with at least 1 NSAID & $3,059(63)$ & $1307(61)$ & $4,366(62)$ & 0.187 \\
\hline Patient with at least 1 knee injection & $1,017(21)$ & $364(17)$ & $1,381(20)$ & $<0.001$ \\
\hline Patient with at least 1 narcotic & $2,336(48)$ & $954(45)$ & $3,290(47)$ & 0.010 \\
\hline Patient with at least 1 other pain meds & $91(2)$ & $36(2)$ & $127(2)$ & 0.595 \\
\hline
\end{tabular}

Values are presented as number (\%).

NSAID: nonsteroidal anti-inflammatory drug.

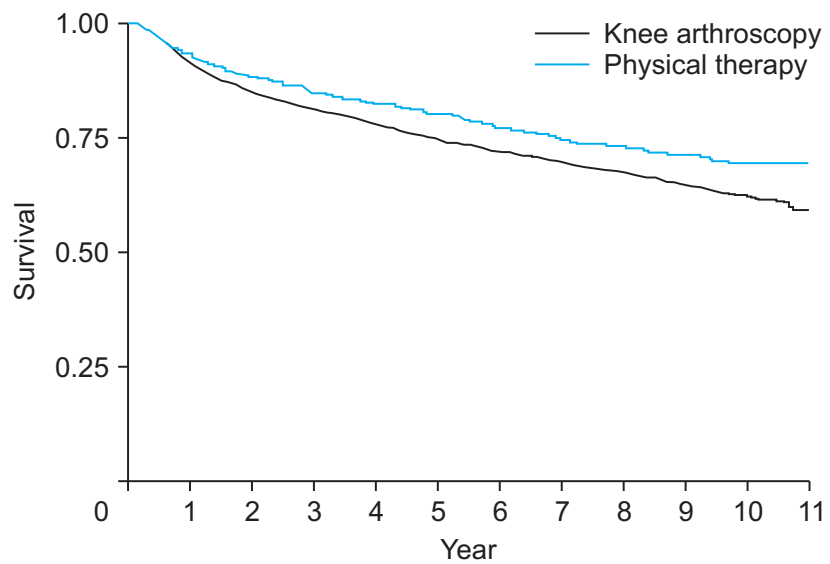

Fig. 2. Survival curves for knee arthroplasty and physical therapy-only groups.

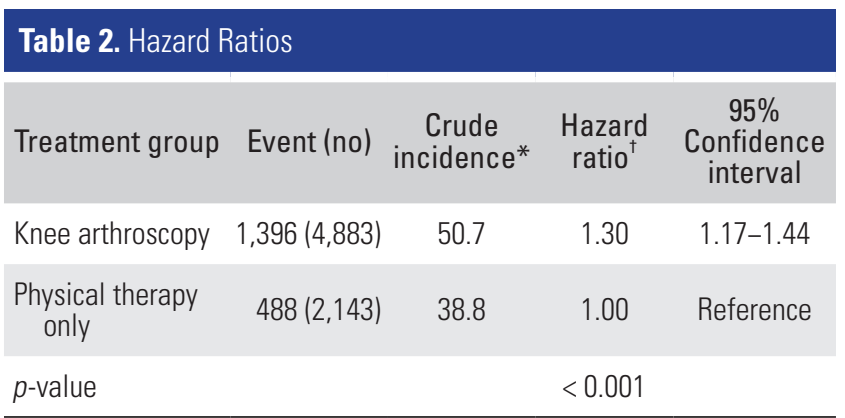

*Per 1,000 person-years. ${ }^{\dagger}$ Weighted, adjusted.

\section{DISCUSSION}

In this study, we demonstrated that in patients with mildto-moderate OA detected on X-ray and MRI-confirmed

\begin{tabular}{|c|c|c|c|}
\hline Variable & $\begin{array}{l}\text { Knee arthroscopy } \\
\quad(n=4,883)\end{array}$ & $\begin{array}{l}\text { Physical therapy } \\
\text { only }(n=2,143)\end{array}$ & $p$-value \\
\hline \multicolumn{3}{|l|}{ Outpatient visit } & 0.93 \\
\hline 0 & 4,420 (96) & 1,959 (96) & \\
\hline 1 & $129(3)$ & $62(3)$ & \\
\hline 2 & $32(1)$ & $16(1)$ & \\
\hline$\geq 3$ & $17(0)$ & $7(0)$ & \\
\hline \multicolumn{3}{|c|}{ Narcotic medication. } & 0.62 \\
\hline 0 & $2,435(53)$ & $1,087(53)$ & \\
\hline 1 & $815(18)$ & $378(18)$ & \\
\hline$\geq 2$ & 1,348 (29) & $579(28)$ & \\
\hline \multicolumn{3}{|l|}{ Knee injection* } & 0.07 \\
\hline 0 & $3,714(81)$ & 1,605 (79) & \\
\hline 1 & $553(12)$ & $262(13)$ & \\
\hline$\geq 2$ & $331(7)$ & 177 (9) & \\
\hline
\end{tabular}

Values are presented as number (\%).

${ }^{*}$ Restricted to those with $\geq 1$ year of follow-up time (95\% of cohort). ${ }^{\dagger}$ Excludes narcotic medications dispensed within 7 days of knee arthroscopy.

meniscal tears, patient characteristics such as older age and greater comorbidity were associated with greater likelihood of treatment with PT-only. Additionally, our data support that among patients with mild-to-moderate OA, those who underwent KA for meniscal treatment were 
$30 \%$ more likely to require partial or total knee replacement or knee fusion at any given time. Additionally, there was no significant difference in clinic encounters for pain management and pharmacy fills for narcotic medications to manage pain symptoms in the first year of follow-up.

Demographic and clinical differences were found between the KA group and PT-only group. Some of these differences could be attributable to clinician judgement, as surgeons may be less likely to recommend surgical treatment to older patients with greater comorbidity burdens. The patients in the PT group also had fewer outpatient visits for knee pain, were more likely to have utilized PT in the year prior to diagnosis, and were less likely to have used narcotics or knee injections. It is possible that this group was already benefiting from PT and decided to continue; whereas the KA group was not given the option of PT, actively chose not to pursue PT, or did not see any benefit from PT. This suggests that there may be different subsets of patients within the group of patients with mildto-moderate $\mathrm{OA}$ and meniscal tears with propensities for KA vs. PT-only.

We also observed a $30 \%$ higher risk of partial or total knee arthroplasty or knee fusion in the group that underwent KA. Though our retrospective study cannot fully determine the cause for this, previous studies have shown an increased risk for degenerative changes in the knee following meniscectomy. ${ }^{18,19)}$ It is thus possible that the meniscal debridement, even if limited, involved in the arthroscopy procedures in our study may be sufficient to disrupt the internal mechanical environment of the knee and hasten the need for total knee arthroplasty. While progression to partial or total knee arthroplasty may be increased, there may still be a specific subset of OA patients that could benefit from KA. For example, OA patients with decreased range of motion due to mechanical blockage from torn meniscal fragments may immediately benefit from intervention with KA through the removal of those fragments. However, this subset of OA patients is still yet to be determined.

In this study, healthcare utilization and medication use in the first year of follow-up for knee pain for patients who underwent KA was not different from that for patients who underwent PT-only. This suggests that undergoing KA did neither alter the amount that patients were seeking care nor utilizing pharmaceutical treatments. Though our study does not directly assess the early pain relief associated with KA, it supplements previous studies that found no difference in outcomes between KA and PTonly. ${ }^{11-13,20-22)}$ While the cohorts in most of these previous studies were patients with minimal OA, ${ }^{11,12,21,22)}$ Katz et al. ${ }^{13)}$ also studied KA versus PT in patients with mild-tomoderate arthritis and found no difference in the amount of improvement 6 months after intervention. Sihvonen et al. ${ }^{20)}$ similarly determined in a multicenter, randomized, sham-controlled trial involving patients with a degenerative medial meniscus tear that arthroscopic partial meniscectomy was not superior to sham surgery, with regards to outcomes assessed during a 12-month follow-up period. Although both groups had significant improvement in all primary outcomes, the patients assigned to arthroscopic partial meniscectomy had no greater improvement than those assigned to sham surgery. These studies were met with criticism from arthroscopists who questioned the authors' methodology. These critics suggested that mechanical symptoms remain an indication for surgery and, if relieved, imply the surgery is of value. ${ }^{23-26)}$ Furthermore, in some of these studies, patients who were originally assigned to the PT group reported benefits after crossing over to the KA group. ${ }^{12,13)}$ Therefore, while KA may not be a good option for every patient, there may be a subset of patients who would benefit from KA rather than just PT alone.

There are limitations to this work that should be addressed. Without utilizing physical examination of the knee, functional status and levels of pain were only indirectly assessed through healthcare utilization. Therefore, potential patient and/or surgeon selection bias due to these factors may not have been specifically addressed. It was also not possible to differentiate between mild, moderate, and severe OA using ICD-9 codes alone. We assumed that patients with a diagnosis of OA who go on to have an MRI and are diagnosed with meniscal tears are individuals with mild-to-moderate arthritis since orthopedic clinical guidelines do not recommend MRI for meniscal damage among individuals with severe arthritis. ${ }^{27)}$ While this assumption was validated with the chart review of 400 patients where the degree of arthritis was examined and assessed, there could have been possible selection bias in that those who had worse OA might be more likely to undergo arthroscopy, and were thus more likely to ultimately need knee arthroplasty or fusion. Furthermore, additional patient factors such as meniscal tear type and lower limb alignment could also contribute to differences between groups but were not collected within this study.

Past research has shown no difference in pain relief and functional status when KA was compared to PT in the setting of knee OA complicated by meniscal tears, and our current study demonstrates that KA does not lengthen the time to further downstream surgery when compared to PT. For patients with meniscal damage complicated by 
Navarro et al. Knee Arthroscopy to Knee Replacement Time

Clinics in Orthopedic Surgery • Vol. 12, No. 3, $2020 \bullet$ www.ecios.org

OA, those who underwent KA were $30 \%$ more likely to have partial or total knee replacement surgery at any given time than those who had PT alone. Our results suggest a reexamination and potential reduction in use of $\mathrm{KA}$ in the setting of knee OA with concomitant meniscal tears when an intended outcome of the KA is to delay eventual TKA.

\section{CONFLICT OF INTEREST}

No potential conflict of interest relevant to this article was reported.

\section{ACKNOWLEDGEMENTS}

This study was supported by a Care Improvement Research Team (CIRT) incubator award made available by Kaiser Permanente Southern California, Department of Research \& Evaluation. Institutional review board guidelines were met during the conduct of this research.

\section{REFERENCES}

1. Felson DT, Naimark A, Anderson J, Kazis L, Castelli W, Meenan RF. The prevalence of knee osteoarthritis in the elderly: the Framingham Osteoarthritis Study. Arthritis Rheum. 1987;30(8):914-8.

2. Jordan JM, Helmick CG, Renner JB, et al. Prevalence of knee symptoms and radiographic and symptomatic knee osteoarthritis in African Americans and Caucasians: the Johnston County Osteoarthritis Project. J Rheumatol. 2007;34(1):17280 .

3. Englund M, Guermazi A, Gale D, et al. Incidental meniscal findings on knee MRI in middle-aged and elderly persons. N Engl J Med. 2008;359(11):1108-15.

4. Sowers M, Karvonen-Gutierrez CA, Jacobson JA, Jiang Y, Yosef M. Associations of anatomical measures from MRI with radiographically defined knee osteoarthritis score, pain, and physical functioning. J Bone Joint Surg Am. 2011;93(3):241-51.

5. Bhattacharyya T, Gale D, Dewire P, et al. The clinical importance of meniscal tears demonstrated by magnetic resonance imaging in osteoarthritis of the knee. J Bone Joint Surg Am. 2003;85(1):4-9.

6. Kim S, Bosque J, Meehan JP, Jamali A, Marder R. Increase in outpatient knee arthroscopy in the United States: a comparison of National Surveys of Ambulatory Surgery, 1996 and 2006. J Bone Joint Surg Am. 2011;93(11):994-1000.

7. Johanson NA, Kleinbart FA, Cerynik DL, Brey JM, Ong KL, Kurtz SM. Temporal relationship between knee arthroscopy and arthroplasty: a quality measure for joint care? J Arthroplasty. 2011;26(2):187-91.

8. Thorlund JB, Hare KB, Lohmander LS. Large increase in arthroscopic meniscus surgery in the middle-aged and older population in Denmark from 2000 to 2011. Acta Orthop. 2014;85(3):287-92.

9. Moseley JB, O'Malley K, Petersen NJ, et al. A controlled trial of arthroscopic surgery for osteoarthritis of the knee. $\mathrm{N}$ Engl J Med. 2002;347(2):81-8.

10. Kirkley A, Birmingham TB, Litchfield RB, et al. A randomized trial of arthroscopic surgery for osteoarthritis of the knee. N Engl J Med. 2008;359(11):1097-107.

11. Herrlin S, Hallander M, Wange P, Weidenhielm L, Werner S. Arthroscopic or conservative treatment of degenerative medial meniscal tears: a prospective randomised trial. Knee Surg Sports Traumatol Arthrosc. 2007;15(4):393-401.

12. Herrlin SV, Wange PO, Lapidus G, Hallander M, Werner S, Weidenhielm L. Is arthroscopic surgery beneficial in treating non-traumatic, degenerative medial meniscal tears? A five year follow-up. Knee Surg Sports Traumatol Arthrosc. 2013;21(2):358-64.

13. Katz JN, Brophy RH, Chaisson CE, et al. Surgery versus physical therapy for a meniscal tear and osteoarthritis. N Engl J Med. 2013;368(18):1675-84.

14. Harris IA, Madan NS, Naylor JM, Chong S, Mittal R, Jalaludin BB. Trends in knee arthroscopy and subsequent arthroplasty in an Australian population: a retrospective cohort study. BMC Musculoskelet Disord. 2013;14:143.

15. Quan H, Sundararajan V, Halfon P, et al. Coding algorithms for defining comorbidities in ICD-9-CM and ICD-10 administrative data. Med Care. 2005;43(11):1130-9.

16. Hirano K, Imbens GW. Estimation of causal effects using propensity score weighting: an application to data on right heart catheterization. Health Serv Outcomes Res Methodol. 2001;2:259-78.

17. Lunceford JK, Davidian M. Stratification and weighting via the propensity score in estimation of causal treatment effects: a comparative study. Stat Med. 2004;23(19):2937-60.

18. Allen PR, Denham RA, Swan AV. Late degenerative changes after meniscectomy: factors affecting the knee after operation. J Bone Joint Surg Br. 1984;66(5):666-71. 
19. Jorgensen U, Sonne-Holm S, Lauridsen F, Rosenklint A. Longterm follow-up of meniscectomy in athletes: a prospective longitudinal study. J Bone Joint Surg Br. 1987;69(1):80-3.

20. Sihvonen R, Paavola M, Malmivaara A, et al. Arthroscopic partial meniscectomy versus sham surgery for a degenerative meniscal tear. N Engl J Med. 2013;369(26):2515-24.

21. Kise NJ, Risberg MA, Stensrud S, Ranstam J, Engebretsen L, Roos EM. Exercise therapy versus arthroscopic partial meniscectomy for degenerative meniscal tear in middle aged patients: randomised controlled trial with two year followup. BMJ. 2016;354:i3740.

22. Yim JH, Seon JK, Song EK, et al. A comparative study of meniscectomy and nonoperative treatment for degenerative horizontal tears of the medial meniscus. Am J Sports Med. 2013;41(7):1565-70.
23. Elattrache N, Lattermann C, Hannon M, Cole B. New England journal of medicine article evaluating the usefulness of meniscectomy is flawed. Arthroscopy. 2014;30(5):542-3.

24. Krych AJ, Carey JL, Marx RG, et al. Does arthroscopic knee surgery work? Arthroscopy. 2014;30(5):544-5.

25. Rossi MJ, D'Agostino RB Jr, Provencher MT, Lubowitz JH. Could the new England journal of medicine be biased against arthroscopic knee surgery? Arthroscopy. 2014;30(5):536-7.

26. Lubowitz JH, Provencher MT, Rossi MJ. Could the New England Journal of Medicine be biased against arthroscopic knee surgery? Part 2. Arthroscopy. 2014;30(6):654-5.

27. American College of Radiology. American College of Radiology ACR Appropriateness Criteria: nontraumatic knee pain. Reston: American College of Radiology; 2012. 